\title{
Philosophiques
}

\section{Agir, intentionnalité et être-au-monde}

\section{Hubert Dreyfus}

Volume 20, numéro 2, automne 1993

Perspectives sur la phénoménologie et l'intentionnalité

URI : https://id.erudit.org/iderudit/027227ar

DOI : https://doi.org/10.7202/027227ar

Aller au sommaire du numéro

Éditeur(s)

Société de philosophie du Québec

ISSN

0316-2923 (imprimé)

1492-1391 (numérique)

Découvrir la revue

Citer cet article

Dreyfus, H. (1993). Agir, intentionnalité et être-au-monde. Philosophiques, 20(2), 285-302. https://doi.org/10.7202/027227ar d'utilisation que vous pouvez consulter en ligne.

https://apropos.erudit.org/fr/usagers/politique-dutilisation/ 


\title{
AGIR, NIENIIONAAIITÉ ET ĖTRE-AL-MONDE
}

\author{
par \\ Hubert Dreyfus
}

La philosophie traditionnelle a maintenu, depuis Platon, que la recherche contemplative et désintéressée constitue notre seule source de connaissance. Depuis Descartes, cette recherche contemplative prétend non seulement définir la nature du sujet et de l'objet de connaissance, mais l'ensemble de l'activité humaine. Selon la tradition, nous pouvons bien sûr porter attention à notre engagement, comme Heidegger le fait dans Êtreet Temps, et arriver à partir de cela à nous considérer comme être-à. Si par contre nous sortons de l'activité engagée pour prendre l'attitude réflexive de l'observateur désintéressé, nous ne pouvons nous concevoir que comme des sujets contemplant des objets. La panoplie de distinctions philosophiques, entre l'expérience subjective interne et l'objet externe de cette expérience, entre l'action de percevoir et le perçu, et entre l'apparence et la réalité, est redevable de cette attitude et « elle n'est plus alors qu'un point de départ "évident" pour les problèmes de théorie de la connaissance ou de la "métaphysique de la connaissance" »I . Pour Heidegger, ce n'est qu'en exposant le caractère dérivé de l'attitude réflexive et désintéressée que nous pouvons voir les limites de la conscience subjective et de sa connaissance des objets.

Pour dépasser cette tradition épistémologique, nous devons partir des phénomènes manifestant la préoccupation quotidienne pour ensuite apercevoir comment la conscience et ses contenus intentionnels peuvent y jouer un rôle. Heidegger soutient que l'expérience [Erfahrung] humaine ouvre le monde et y découvre des entités - ceci n'entraînant pas la conclusion traditionnelle voulant que les êtres humains se rapportent aux objets par leurs vécus [Erlebnisse], c'est-à-dire par des états mentaux. Cette

\footnotetext{
I. Martin Heidegger, Être et temps, Paris, Gallimard, 1990, p. 93 [Sein und Zeit, p. 59 l.
} 
perspective défie le sens commun et une longue tradition philosophique.

Être-parmi, en tant qu'existential, est bien sûr un problème en lui même. Ce problème est précisément dû à l'apparente évidence posant comme premisse la relation sujet-objet. Il est remarquable que ce problème ne puisse être évité. Il est aussi vieux que la philosophie et apparaît déjà chez Parménide. L'explicitation, développée originairement et de prime abord dans la compréhension pré-philosophique du Dasein, de l'âme comme pensée et représentation, comme conscience, établit une relation aux objets, ou pour le dire autrement, les étants apparaissent opposées à la pensée, à l'intuition, et à la représentation $[. . .]^{2}$.

L'opposition de Heidegger à cette position traditionnelle peut être rapprochée de celles de Michael Polanyi ${ }^{3}$ et de Thomas $\mathrm{Kuhn}^{4}$. Ces trois penseurs prétendent que la connaissance théorique et désintéressée, correctement décrite dans les termes d'une relation sujet / objet et présentée depuis 2500 ans comme le meilleur exemple de connaissance, présuppose un « savoir-faire » pratique et impliqué dont on ne peut rendre compte par ces mêmes termes. D'après ces penseurs, la connaissance théorique repose sur des habiletés pratiques. (Ceci n'implique évidemment pas que l'objet découvert par la réflexion théorique repose sur ces habiletés, même si Kuhn, contrairement à Heidegger, semble tirer cette conclusion $)^{5}$.

\section{la critique heideggerienne de l'intentionnalité}

La différence fondamentale entre Heidegger et la tradition de la recherche désintéressée, tradition qui atteint son point culminant avec Husserl, est évidente si on se fie aux exemples choisis par chacun. Husserl parle, à la suite de Kant, de la « synthèse » mentale requise pour recevoir la succession des expériences visuelles d'un dé lorqu' on se déplace autour de celui-ci ${ }^{6}$. C'est sur la base de cette synthèse qu'il est possible de percevoir ce dé comme un objet perdurant, et de lui donner aussi la signification de « quelque chose à lancer ». Ceci démontre, selon Husserl, que

2. Martin Heidegger, Metaphysische Anfangsgründe der Logik im Ausgang von Leibniz, Gesamtausgabe Bd 26, Francfort, V. Klostermann, 1978, p. 162.

3. Michael Polanyi, Personal Knowledge, Londres, Routledge and Kegan Paul, 1962, ch. 4.

4. Thomas Kuhn, La Structure des Révolutions Scientifiques, Paris, Flammarion, 1983.

5. Pour une discussion complète de ce problème, voir Hubert L. Dreyfus, Being-in-theWorld, Cambridge Mass., M.I.T. PTess, 199I, ch. I5.

6. Edmund Husserl, Méditations Cartésiennes, Paris, Vrin, I986, p. 34. 
de « quelque chose à lancer ». Ceci démontre, selon Husserl, que la perception et l'action impliquent nécessairement l'activité mentale.

Heidegger prend comme exemple l'agir engagé dans le monde et nécessitant l'usage d'outils comme des marteaux et des poignées de portes. Il tente de démontrer que ce qui est ainsi révélé se trouve à l'opposé de ce que Descartes et Husserl prétendaient. Plutôt que de percevoir tout d'abord des aspects, pour ensuite les synthétiser en objets et finalement assigner une fonction à ceuxci en se basant sur leurs propriétés physiques, nous manipulons habituellement des outils ayant déjà une signification dans un monde structuré en termes de finalité. Pour bien voir cela, nous devons d'abord dépasser l'interprétation traditionnelle suivant laquelle la théorie précède la pratique. Alors seulement pourronsnous décrire notre rapport pratique et engagé avec les choses ainsi que ce qu'il révèle. Une nouvelle approche phénoménologique est donc requise.

I'étant phénoménologiquement préthématique, en l'occurrence ce dont il est fait usage [...] devient accessible si on se transfère dans une préoccupation de cet ordre. En toute rigueur, parler ainsi de transfert risque d'égarer ; car ce genre d'être où le commerce est notre préoccupation, nous n'avons pas à commencer par nous y transférer. Toujours le Dasein quotidien est déjà de cette manière, par exemple : pour ouvrir la porte, je fais usage de la poignée. Pour gagner l'accès phénoménologique à l'étant qui se rencontre ainsi, il s'agit plutôt d'arriver à contenir l'affluence et la confluence des tendances à expliciter qui dissimulent entièrement le phénomène d'une telle « préoccupation » et n'ont pas de meilleur moyen de le faire qu'en dissimulant justement l'étant tel qu'il se rencontre de luimême dans cette préoccupation et pour elle?

Une version extrême de l'attitude à laquelle Heidegger s'oppose apparaît dans le roman La Nausée de Sartre, dont le personnage principal, Roquentin, succombe jusqu'à la psychose au traditionnel « désintéressement ». Roquentin perçoit une poignée de porte-Sartre utilise aussi cet exemple-comme un objet de métal, froid, touchant la paume de sa main. Sartre pense (comme Descartes et Husserl) que son héros retourne, par son extrême désintéressement, à la pure perception de l'être fondamental des choses.

7. $\hat{E} T$, op. cit., p. $\mathrm{IO}_{3} ;$ SuZ, p. 67. 
qu'il ferait de la signification philosophique du cas étudié par Sartre serait complètement différente. Contrairement à Descartes, Husserl et Sartre, Heidegger considère l'objet de la simple observation non pas comme ce qui est réellement, mais comme un résidu appauvri de l'outillage directement manipulé. Les simples objets d'une pure perception désintéressée ne sont pas des choses simplement données que nous pourrions subséquemment utiliser, mais plutôt les débris de notre monde pratique et quotidien que nous laisse l'inhibition de l'action.

C'est comme si Heidegger inversait ainsi la tradition et considérait la contemplation désintéressée comme une modification privative de l'engagement quotidien. Il semble dire que le sujet désintéressé, connaissant et conférant la signification, qui est au centre de la phénoménologie husserlienne, doit être remplacé par un sujet corporel, agissant et conférant la signification. Mais si l'on inverse sans plus la tradition, on risque d'être mécompris et ainsi de faire l'objet d'une (rélappropriation. En effet, Dagfinn Føllesdal a sous-estimé l'originalité de Heidegger précisément sur ce point. Dans son article sur le rôle de laction chez Husserl et Heidegger, il attribue à ce dernier l'interprétation suivant laquelle Husserl aurait surestimé la contemplation détachée, et il défend ce qu'il estime être la contribution principale de Heidegger, à savoir que l'activité pratique corporelle est le mode fondamental par lequel le sujet prête sens aux objets.

On a souvent prétendu que l'activité pratique présuppose une compréhension théorique du monde I...I. Heidegger rejette cela. Il considère que les différentes manières pratiques d'être en rapport avec le monde sont plus fondamentales que les modes théoriques. l...] je considère l'idée de Heidegger voulant que toute l'activité humaine joue un rôle dans notre constitution du monde, ainsi que son analyse de ce phénomène, comme ses principales contributions à la philosophie ${ }^{8}$.

8. Dagfinn Follesdal, « Le rôle de l'action dans la constitution du monde chez Husserl et Heidegger » dans ce numéro, p. $275^{-276}$, un première version de ce texte est parue dans E. Saarinen et al., (dir.), Essays in Honour of Jaakko Hintikka, Dordrecht, Reidel, 1979. p. 37I. Une réduction similaire des travaux de Heidegger à une version pratique de la pensée de Husserl est réalisée par Mark Okrent, Heidegger's Pragmatism, Ithaca, Comell University Press, 1988. Okrent dit camément : « Aussitôt que lon réalise que pour Heidegger, l'intentionnalité est toujours pratique plutôt que cognitive et que la forme primaire de lintention consiste à faire quelque chose par rapport $\dot{a}$ un but plutôt quáa être conscient de quelque chose, les analogies structurelles entre les stratégies argumentatives de Husserl et de Heidegger deviennent évidentes » (p. 1o). 
Føllesdal rapporte que :

1...] après son arrivée à Freibourg en 1916, plus précisément vers la fin des années dix et au début des années vingt, il est clair que Husserl devint alors de plus en plus conscient du rôle important joué par l'activité pratique dans notre rapport au monde

[...]

Il s'agit là, pour Husserl, d'« une suite infinie de buts, d'objectifs, et de tâches $\gg$ auxquelles se rapportent nos actions ainsi que leurs produits 9 .

Føllesdal se propose de déterminer à qui est redevable ce nouvel intérêt pour la phénoménologie de l'activité pratique :

[...] que ce dernier avait des idées similaires à celles de Heidegger, et ce bien avant la publication de Être et Temps. Ces idées prirent racine chez Husserl, peu après son arrivée à Freiburg où il rencontra Heidegger en Igi6. Il est possible aue Husserl ait attire Heidegger [...]. Cependant, il est également possible que Husserl ait été attiré dans cette direction suite à des discussions avec le jeune Heidegger ${ }^{\text {Io }}$.

Une fois qu'on aperçoit l'écart profond qui sépare Heidegger de Husserl et de la tradition, on réalise que la question soulevée par Føllesdal, bien qu'interessante, est hors de propos. C'est l'intentionnalité qui est le véritable enjeu. Tel qu'utilisé par Franz Brentano et ensuite par Husserl, le terme « intentionnalité » définit le fait que des états mentaux, tels que percevoir, croire, désirer, craindre, et avoir une intention (pris au sens courant), se réfèrent toujours à quelque chose. Ils sont toujours dirigés vers un certain objet sous une certaine description, peu importe que cet objet existe ou non à l'extérieur de ces états mentaux. Le contenu intentionnel ou représentationnel de ces états est la caractéristique mentale rendant possible cette directionalité. En orientant la discussion sur l'importance relative de l'activité engagée et de la contemplation désintéressée, Føllesdal néglige l'argument le plus radical de Heidegger, argument selon lequel l'explication traditionnelle de ces deux modes d'être en rapport au monde présupposent, sans en rendre compte, une intentionnalité plus fondamentale.

[Être-à] est tout autre chose qu'une prise de position dans un visà-vis où il ne s'agit que de contemplation ou d'action, c'est-à-dire qu'une réunion là-devant d'un sujet et d'un objet ${ }^{\mathrm{II}}$.

9. Ibid, p. $276-77$.

10. Ibid., P. $28 \mathrm{z}$.

II. $\hat{E} T$, p. $224 ; S u Z$ p. 176 . 
Heidegger ne considère pas que l'activité pratique est première; il veut plutôt montrer (avec tout le respect dû à Husserl) que ni l'activité pratique, ni la connaissance contemplative ne peuvent être comprises comme une relation entre un esprit autonome et un monde indépendant. Il est cependant naturel que Føllesdal n'ait pas reconnu la véritable originalité de Heidegger puisque ce dernier soutient que l'attitude engagée, avec ce qu'elle révèle, est en quelque sorte plus originaire que l'attitude désintéressée et ce que celle-ci révèle - c'est-à-dire, comme l'indique le titre de la section $\mathrm{r} 3$, « Exemplification de l'être-à [...] à partir d'un mode dérivé : la connaissance du monde », connaître le monde est un mode fondé d'être-à. Le connaître étant exemplaire de la relation sujet / objet, de considérer que la connaissance est fondamentale nous limite dès le départ à l'image intentionnaliste d'un être humain en tant que sujet de croyances (justifiées ou non) à propos d'objets et d'états de choses. La stratégie de Heidegger consiste, premièrement, à inverser les priorités habituelles. Mais cette inversion de la priorité du connaître sur l'agir [doingl ne fait que préparer le terrain à la question phénoménologique : quel est le mode d'être de l'intentionnalité?

Il appartient à l'essence de la personne qu'elle n'existe que dans l'accomplissement d'actes intentionnels [...]. Mais quel est le sens ontologique d'《 accomplir $»^{12}$ ?

La conception traditionnelle de l'agir est, du moins depuis Descartes, représentationnelle. Des philosophes contemporains comme John Searle et Donald Davidson s'entendent, contrairement à leur habitude, sur la nécessité de décrire les actions en termes de désirs et de croyances, c'est-à-dire d'états mentaux, lesquels causent des mouvements corporels. L'entreprise de Heidegger visant à en finir avec la tradition repose principalement sur sa tentative de dépasser la distinction sujet / objet dans tous les domaines, y compris dans celui de l'action. Dans un cours, il écrit :

Mon intention essentielle est premièrement de poser le problème (de la relation sujet / objet) pour ensuite m'y pencher de telle sorte que l'essentiel de la tradition occidentale puisse être concentrée en un problème simple et fondamental ${ }^{\mathrm{I}}$.

Le problème principal ne concerne donc pas la priorité d'un type d'intentionnalité - théorique ou pratique - sur un autre,

12. ÊT, op. cit., p. 80 ; SuZ, p. 48.

13. Metaphysische Anfangsgründe, op. cit., p. 165. 
mais plutôt sur la possibilité de dépasser la manière traditionnelle de rendre compte de l'intentionnalité comme telle.

Déjà dans son cours de ig25 - deux ans avant Être et Temps Heidegger questionne ces présupposés afin de les dépasser et d'arriver à miner la priorité de la relation sujet / objet sous toutes ses formes :

IL'intentionnalitél n'est pas une explication ultime du psychique, mais une approche initiale permettant d'éliminer l'application non critique de réalités définies traditionnellement telle que le psychique, la conscience, la continuité de l'expérience vécue et la raison ${ }^{\mathrm{T}}$.

Tout tourne ainsi autour de la critique heideggerienne de la théorie husserlienne de l'intentionnalité. Comme l'écrit Heidegger :

Ici encore, nous avons un terme et un concept allant tellement de soi que l'on ne s'y attarde plus et que l'on tient, même à un stade préparatoire, pour la solution du problème, comme s'il s'agissait sans plus de la clé ouvrant toutes les portes. Au contraire, c'est ce qui est par là même visé qui doit être problématisé ${ }^{15}$.

L'objection de Heidegger ne vise pas l'introduction dans l'esprit, par la théorie de l'intentionnalité, d'une image qui apparaîtrait entre le sujet et l'objet. Husserl rejette explicitement cette idée. Pour comprendre l'enjeu qui entoure la question de l'intentionnalité, il faut savoir qu'à ses débuts, Husserl, comme Searle, maintenait une notion minimale de représentation et de contenu intentionnel. L'esprit n'est pas dirigé vers une sorte d'objet contenu en lui et qui serait de plus reflété dans le monde. Parler en termes de contenu intentionnel permet de saisir le fait que les perceptions, les croyances, les désirs, et les intentions peuvent tous être dirigés vers le même objet sous le même aspect. Par exemple, je peux percevoir que je me rends au travail, croire que je me rends au travail, désirer me rendre au travail, avoirl'intention de me rendre au travail, et ainsi de suite.

Cependant, Heidegger voit bien qu'une telle explication introduit déjà la distinction sujet / objet. Elle admet la séparation entre un contenu intentionnel mental et un monde objectif pouvant ou non être tel que l'esprit le conçoit. Husserl définit la phénoménologie comme étant l'étude du contenu intentionnel

14. Prolegomena zur Geschichte des Zeitbegriffs, Gesamtausgabe Bd 20, Francfort, V. Klostermann, 1979, p. 63 ; tr. fr. J.F. Courtine, Les problèmes fondamentaux de la phénoménologie, Paris, Gallimard, 1985 , p. 69.

15. Metaphysical Foundation of Logic, op.cit., p. 166. 
qui reste dans l'esprit suite à la mise entre parenthèses du monde $\mathrm{e}^{\mathrm{I}}$. Heidegger accepte que la directionalité intentionnelle soit essentielle à l'activité humaine, mais il refuse par contre de lier l'intentionnalité au mental, et donc qu'elle soit, telle que Husserl le prétend (à la suite de Brentano), la caractéristique distinctive des états mentaux.

La conception courante de l'intentionnalité [...] méconnaît du même coup la structure du se-diriger-sur, l'intentio. La mécompréhension réside dans une subjectivisation à contresens de l'intentionnalité. On pose un Moi, un sujet, à la « sphère » duquel appartiennent des vécus intentionnels l.... L'idée d'un sujet n'ayant de vécus intentionnels que dans sa sphère l...] mais restant enfermé dans sa boîte, est un non-sens qui méconnaît la structure ontologique fondamentale de l'étant que nous sommes nous-mêmes ${ }^{17}$.

Pour développer une véritable ontologie, Heidegger utilise son propre terme, soit Verhalten, que je traduis par « comportement $\gg$ [comportment], afin de définir la manière dont l'être humain se rapporte aux choses.

Les comportements ont la structure du se-diriger-sur, de l'êtrecentré et orienté-sur. La phénoménologie, en s'appuyant sur un terme scolastique, caractérise cette structure comme intentionnalite $e^{18}$.

En référence à notre activité dirigée vers quelque chose, Heidegger emploie le terme « comportement » précisément parce que celui-ci ne véhicule aucune connotation mentaliste. Il tient toute cette mécanique du mental pour une construction des théoriciens, et non pour le résultat d'une description phénoménologique. Il considère ainsi le comportement, ou l'intentionnalité, comme étant caractéristique non seulement des actes de la conscience, mais de l'activité humaine en général. L'intentionnalité n'est pas attribuée à la conscience, mais au Dasein.

Dans la mesure où la séparation couramment opérée entre un sujet, avec sa sphère immanente, et un objet avec une sphère transcendante - de manière encore plus générale, la différence entre un dedans et un dehors - est construite de toutes pièces et fournit toujours occasion à de plus amples constructions, nous ne parlerons plus à l'avenir de sujet, ni de sphère subjective, mais nous

16. Pour une explication de la réduction phénoménologique de Husserl, par exemple sur la manière dont Husserl et Searle ont été conduits, par leur théorie du contenu intentionnel, à ce que Jerry Fodor a nommé le « solipsisme méthodologique », voir mon introduction à Husserl, Intentionality, and cognitive Science, H. Dreyfus, (dir.), op. cit.

17. Problemes fondamentaux, op. cit., p. 88-89.

I8. Ibid., p. 82, nous avons ajouté les premiers italiques. 
comprendrons l'étant auquel appartiennent ces comportements intentionnels comme Dasein, et cela de manière à essayer de caractériser comme il convient l'être du Dasein - une de ses structures fondamentales - à l'aide du comportement intentionnel bien compris ${ }^{19}$.

Heidegger souligne que lintroduction par Husserl du contenu intentionnel - une structure idéale supposée ni physique, ni psychique - dans le but d'expliquer la directionalité de la conscience et de combler ainsi l'écart entre le sujet et l'objet, soulève plus de problèmes qu'elle n'en résout.

Maintenant plus on s'attache à cette idée simple que le connaitre est d'emblée et par nature « interne » mais qu'il n'a aucunement le genre d'être d'un étant physique et psychique, d'autant plus croit-on avancer sans présupposition dans la question de l'essence de la connaissance et dans l'élucidation du rapport entre sujet et objet [...]. Mais de quelque manière que puisse être explicitée cette sphère intèrieure, $s^{\prime} i l$ arrive que soit au moins posée la question de savoir comment le connaître parvient à s'en « extraire » pour atteindre une « transcendance », tout ce qu'on voit c'est qu'on trouve le connaitre problématique sans avoir auparavant clarifié une bonne fois comment est et ce qu'est le connaître qui pose de telles énigmes ${ }^{20}$. Il apparaît très clairement à Heidegger que la priorité accordée par Husserl à la connaissance ne peut être sans plus augmentée par l'action. Il s'agit plutôt de réviser entièrement cette idée de transcender de l'intérieur vers l'extérieur. Heidegger critique la façon traditionnelle de rendre compte de lintentionnalite quotidienne, qu'il appelle « transcendance ontique », puisqu'elle néglige un mode d'être plus fondamental.

Nous devons faire de l'intentionnalité elle-même un problème. L'intentionnalité se rapporte en fait aux étants eux-mêmes, et est en ce sens un comportement ontique transcendant; mais ce dernier ne détermine pas originairement ce rapport-à, il est plutôt fondé sur l'être-parmi les étants. Cet être-parmi, dans sa possibilité intrinsèque, est à son tour fondé dans l'existence. De cette façon, la limitation de l'interprétation et de la fonction utilisée jusqu'à maintenant du concept d'intentionnalité, ainsi que sa signification fondamentale, deviennent clairs ${ }^{21}$.

Heidegger soutient que toute relation d'états mentaux à leurs objets présuppose une forme plus fondamentale d'être-avec-leschoses n'impliquant aucune activité mentale.

\footnotetext{
19. Ibid., p. 89.

20. ÊT, op. cit., p. 94-95; SuZ, p. 6o-61.

21. Metaphysische Anfangsgründe, op. cit., p. 168.
} 
L'intentionnalité appartient à l'existence du Dasein [...]. Exister signifie donc aussi être auprès de l'étant en se comportant par rapport à lui. Il appartient à l'essence du Dasein d'exister de telle sorte qu'il est toujours déjà auprès d'un étant autre que lui ${ }^{22}$.

Sa façon de comprendre le Dasein permet à Heidegger de saisir pourquoi, traditionnellement, la connaissance a été considérée de façon inexacte comme étant fondamentale, et pourquoi même l'action a été interprétée comme étant une sorte de connaissance.

[L'existencel apporte non seulement une modification des concepts traditionnels de conscience et d'esprit; la formulation radicale de l'intentionnalité du phénomène dans une ontologie du Dasein entraine un dépassement fondamental et « universel » de cette position. A partir de là, le concept précédent d'intentionnalité apparait comme une conception restreinte l.... A cause de cette restriction, l'intentionnalité est conçue fondamentalement comme « viser »...l. Ainsi, chez Husserl par exemple, tout acte consistant à s'orienter vers quelque chose est caractérisé comme étant une connaissance ${ }^{23}$.

Cette dernière citation montre que dans ses cours, un an après la publication d'Être et Temps, Heidegger sent le besoin d'expliquer que ce à quoi tendent les sections i2 et i3 va bien au delà du renversement de la priorité du connaître sur l'agir [doingl. Il est profitable de citer en entier sa nouvelle interprétation de ces sections plutôt déroutantes, puisqu'il s'agit de l'explication la plus explicite que donne Heidegger sur le point fondamental de la division I d'Être et Temps.

Sous-jacent au problème antérieur de la « relation » de « sujet »à «objet », se trouve le problème encore ignoré de la transcendance l..... Le problème de la transcendance comme telle est absolument différent du problème de l'intentionnalité. En tant que transcendance ontique, cette dernière n'est elle-même possible que sur le fondement de la transcendance originaire, sur le fondement de l'être-aumonde. Cette transcendance primaire rend possible toute relation intentionnelle aux étants [...]. La relation est fondée sur une précomprehension de l'être des étants. Cette comprehension de l'être assure premièrement la possibilité aux étants de se manifester euxmêmes en tant qu'étants ${ }^{24}$.

Une des principales tâches préparatoires d'Être et Temps consiste à mettre en lumière l'essence originaire de cette « relation $»[. . .]^{25}$.

Heidegger revient de nouveau, lors de ces cours, sur ces mêmes sections d'Être et Temps. En attirant l'attention sur ce qui

22. Problemes fondamentaux_, op. cit., p. 195.

23. Metaphysical Foudation of Logic, op. cit., P. I68-169, nous soulignons.

24. Ibid., p. 169-170.

25. Ibid., p. i64. Cf. sections 12 et 13 comme premières caractérisations préparatoires. 
est plus fondamental que tout état intentionnel, que celui-ci représente le monde tel qu'il est (croyance) ou tel que l'on voudrait qu'il soit (désir), Heidegger essaie d'éviter la mécompréhension de Husserl et de Follesdal voulant qu'il renverse simplement la priorité de l'intentionnalité théorétique sur l'intentionnalité pratique.

La tâche centrale de l'ontologie du Dasein est de revenir en deçà de ces divisions au sein des comportements afin de trouver leur racine commune, une tâche qui ne doit pas, bien sûr, être facile. La transcendance originaire précède tout mode possible d'activité en général, antérieurement à la noésis Icroyancel, mais aussi antérieurement à l'orexis $\mid$ désir $]^{26}$.

Ou comme il l'exprime dans son cours de I927:

Quelle que soit la manière dont on appréhende le connaitre [...] celui-ci définit un comportement vis-à-vis de létant l.... Mais tout commerce pratico-technique avec l'étant est également un comportement vis-à-vis de l'ètant [...]. Dans tout comportement vis-à-vis de l'étant, qu'il s'agisse spécifiquement du connaître que l'on caractérise en général comme théorique, ou qu'il s'agisse de la connaissance pratico-technique, la compréhension de l'être est déjà présente. En effet, c'est seulement à la lumière de la compréhension de l'être que l'étant peut nous rencontrer en tant que tel ${ }^{27}$.

Ainsi, dès I928, l'entière signification du rejet par Heidegger de toute forme purement mentaliste de l'intentionnalité est pleinement explicite, tout comme l'est sa raison de commencer par ce qui ressemble à tort à un renversement de priorité entre deux relations sujet / objet, la connaissance et l'agir.

Dans la mesure où le Dasein existe comme être-au-monde, il est dejaj dehors parmi les étants ; même si cette façon de parler est encore imprécise puisque « déjà dehors » présuppose que le Dasein ait déjà pu être à l'intérieur. Même si je dis que l'activité intentionnelle du Dasein est toujours déjà ouverte sur les étants et pour les étants, la présupposition qu'elle ait déjà étē fermée est encore sousjacente. Ce que nous entendons par transcendance ne peut être rendu compatible avec les formulations qu'on en a données jusqu'à maintenant, et il est très difficile à apercevoir à la lumière de cette habituelle version claquemurée du problème. Ni Bergson l... ni Husserl ne voit le problème et le phénomème [...]. Nous devons essayer de clarifier cette constitution fondamentale en partant premièrement du concept traditionnel de la relation épistémologique sujet / objet. Ainsi la recherche que constitue Être et Temps, après les explications préliminaires et le premier chapitre, commence par :

26. Ibid., p. $235^{-2} 236$.

27. Problèmes fondamentaux, op. cit., p. 331. 
« L'être-au-monde en général comme constitution fondamentale du Dasein » et les sections i2 et i3 présentent une esquisse et une première introduction au phénomène ${ }^{28}$.

Ainsi les sections I2 et I3 sont censées montrer qu'en partant d'états intentionnels, réceptifs ou actifs, même si ces états mentaux sont tacites ou préréflexifs, nous déformons le phénomène de la préoccupation quotidienne et sommes repoussés au sein des vieux problèmes épistémologiques. Donc, avant de soulever la question du statut phénoménologique et ontologique de la conscience, il est nécessaire de réinterpréter le mode quotidien qu'a le Dasein d'être-à. L'analyse par Heidegger de la situation naturelle dans laquelle se déroulent les activités quotidiennes doit montrer que la position épistémique traditionnelle d'un esprit distinct des objets, qu'il les observe ou qu'il agisse sur eux, est un mode déficient d'être-au-monde et ne peut donc avoir les implications philosophiques essentielles qu'ont supposées les philosophes modernes de l'esprit.

\section{La pertinence actuelle de la critique heideggerienne}

Avant de pouvoir pleinement évaluer la difficulté du projet de Heidegger et de décider s'il réussit à le mener à terme, nous devons préciser le mieux possible la théorie intentionnelle de l'esprit développée par Husserl, théorie à laquelle il s'oppose. Comment la distinction sujet / objet est-elle censée être constituée au sein de toutes les manières de se rapporter au monde, que cellesci relèvent de la connaissance ou de l'agir ? Etant donné que Heidegger concentre ses recherches sur l'action en tant que domaine illustrant le mieux l'idée que notre expérience n'implique pas nécessairement une distinction esprit / monde, je me pencherai sur une explication de l'action. Puisque Husserl n'a jamais développé de théorie de l'action, je me tournerai vers John Searle qui défend une formulation détaillée et convaincante de l'explication intentionnaliste à laquelle Heidegger s'oppose. Pour être en mesure d'apprécier la force et l'originalité de la charge de Heidegger contre l'intentionnalité mentaliste, autour de laquelle tout le reste de la problématique gravite, je dois tout d'abord exprimer la formulation que Searle donne de la façon dont la distinction esprit / monde est constituée dans l'expérience de l'agir.

28. Metaphysische Anfangsgründe, op. cit., p. 213-214. 
Les philosophes analytiques s'entendent généralement sur le fait que nos concepts communs de perception et d'action sont des concepts causaux. Paul Grice a montré que notre concept de perception nécessite une expérience causée d'une façon déterminée par l'objet perçu ${ }^{29}$. Dans le domaine parallèle de l'agir, Searle et Davidson s'accordent pour dire que notre concept d'action est également causal - c'est-à-dire qu'une action consiste en un mouvement corporel causé d'une façon déterminée par un état mental. Davidson pense que ce mouvement doit être causé par une croyance et par un désir ; Searle défend une position plus minimale qui prétend seulement qu'il doit être causé par une intention.

Searle distingue deux types d'intentions causant les actions : les intentions préalables et les intentions en action. Comme ces termes le suggèrent, l'intention préalable se forme antérieurement à l'action, alors que l'intention en action est simplement simultanée à l'action. Searle écrit : « Toutes les actions intentionnelles ont une intention en action, mais toutes n'ont pas d'intention prealable ${ }^{30}$. $\gg$ Des actions spontanées (sautiller et faire les cent pas tout en pensant est l'exemple favori de Searle) n'impliquent pas la formation d'une intention préalable, mais doivent néanmoins être causées par une intention en action. Searle prétend que tant l'intention préalable que l'intention en action sont causalement auto-référentielles, du fait qu'elles contiennent dans leurs conditions de satisfaction l'exigence que l'intention soit la cause de ses propres conditions de satisfaction. (Les conditions de satisfaction définissent selon Searle ce qui équivaut au succès d'un état intentionnel, c'est-à-dire ce qui équivaut à la satisfaction d'un désir, à la vérité d'une assertion, à la véracité d'une perception, etc.) Dans le cas de l'action, les conditions de satisfaction représentent plus que l'accomplissement d'un quelconque mouvement corporel. Si les conditions de satisfaction adviennent, c'est-à-dire si le corps bouge, mais que cela n'est pas causé d'une façon déterminée, l'intention n'a pas été satisfaite. L'auto-référentialité renvoie à l'inclusion de l'exigence causale voulant que l'intention soit la cause du mouvement, et ce au sein du contenu de

29. Paul Grice, "The Causal Theory of Perception », dans Robert Swartz, (dir.), Perceving, Sensing, and Knowing, New York, Anchor Books, 1965.

30. Searle, L'intentionnalité, Essai de philosophie des états mentaux, Paris, Editions de Minuit, I985, p. 109 
l'intention lui-même. En bref, une action est un mouvement corporel causé par l'intention de l'accomplir.

Selon Searle, l'intention en action constitue l'expérience de l'agir - ce que William James nomme « le sentiment d'effort » Ifeeling of effortl. James écrit :

Que nous ayons un sentiment d'effort, cela ne fait aucun doute. Le langage populaire a suffisament consacré ce fait par l'institution du mot effort, ainsi que de ses synonymes : s'évertuer lexertionl, tâcher Istrivingl, peiner [strainingl. La différence entre une simple sensation passive et celle qui contient les éléments de la volition et de l'attention a été également retenue dans le discours populaire par la différence établie entre certains verbes tels que voir et regarder ; entendre et écouter ; sentir (to smell) et humer Ito scentl; ressentir et toucher ${ }^{3 \mathrm{I}}$.

D’après l'analyse de Searle, tout agir est accompagné d'une expérience d'agir, et cette expérience d'effort a pour contenu intentionnel d'être la cause de mes mouvements corporels. L'expérience mentale d'agir et le mouvement physique appartiennent à des domaines totalement séparés. Je peux faire l'expérience d'agir même si je me trompe - par exemple, si je suis paralysé - et que le mouvement corporel n'a pas lieu. Ainsi, selon Searle, la distinction entre esprit et monde, entre sujet et objet, est directement constituée aussi bien dans le concept d'action que dans la phénoménologie de l'agir.

Comme nous l'avons vu, James associe attention, volition et effort (ce qui correspond à l'expérience de l'agir chez Searle). Nous faisons souvent l'expérience de nous-mêmes comme d'un sujet conscient et de nos efforts conscients comme s'ils étaient la cause de nos actions. En fait, Heidegger admettrait que la tradition philosophique a érigé l'auto-référentialité à travers notre concept de conscience. Lors d'un séminaire, il demande « Mais où dans la philosophie commence la conscience? $\gg$ Et il répond :

Avec Descartes, toute conscience de quelque chose est à la fois conscience de soi [...l. Il n'y a pas de conscience sans conscience de soi, ce qui ne veut toutefois pas dire que le soi doive devenir thématique. Il s'agit là de la structure la plus universelle de la représentation ou, au sens husserlien, d'une conscience de quelque chose $^{32}$.

31. William James, «The Feeling of Effort 》, dans les Collected Essays and Reviews, New York, Longmans, Green and Co., 1920, p. 151-152.

32. Heidegger, Zollikonner Seminare, Frankfurt, V. Klostermann, 1987, P. 284-285. 
Heidegger pourrait bien aussi admettre que James et Searle ont décrit avec précision l'expérience auto-référentielle de l'action délibérée et que rendre compte d'une façon formelle du contenu intentionnel d'une telle experience, comme le fait par exemple Searle, constitue un champ légitime pour l'analyse phénoménologique. Heidegger reconnaît qu'il nous est possible de décrire la conscience auto-référentielle comme le fait Husserl dans sa phénoménologie:

Peut-être cette manière de se donner qu'offre la simple, formelle, réflexive perception du je est-elle effectivement évidente. Il y a même dans cette connaissance un accès ouvrant à une problématique phénoménologique autonome qui a, en tant que « phénoménologie formelle de la conscience $»$, sa signification fondamentale et son cadre $\mathrm{e}^{33}$.

Mais il s'empresse de demander, « Va-t-il donc a priori de soi que l'accès au Dasein doive être une réflexion simplement percevante sur le je des actes ${ }^{34}$ ? »

Bien sûr, une telle conscience auto-référentielle ne constitue pas l'enjeu d'Être et Temps. Selon Heidegger, une telle conscience est un mode spécifique et dérivé du dévoilement. Ce n'est pas toute l'activité humaine qui est délibérée, et ainsi, ce n'est pas toute l'activité qui est causée par un état mental auto-référentiel. Nietzsche vit le même phénomène:

Nous pourrions en effet penser, sentir, vouloir, nous ressouvenir, nous pourrions de même « agir » dans tous les sens du terme : tout ceci cependant n'aurait nullement besoin d' «entrer dans notre conscience » (comme on dit de façon imagée). La vie toute entière serait possible sans pour autant se voir réfléchie : c'est effectivement ainsi d'ailleurs que pour nous la majeure partie de la vie continue à s'écouler sans pareille réflexion - y compris même notre vie pensante, sensible, voulante ${ }^{35}$.

Cependant, comme le suggère Searle, il semble y avoir une sorte de conscience de soi dans l'action, étant donné que si on s'arrête et questionne quelqu'un agissant de façon non délibérée, il est tout de même en mesure de dire ce qu'il est en train de faire. Ceci démontre, pour Searle, que même dans le cas d'une telle activité non délibérée, nos mouvements sont guidés par une intention en action auto-référentielle. Heidegger, comme

33. ÊT, op.cit., p. I57; SuZ, p. 115 .

34. Ibid.

35. Friedrich Nietzsche, Le Gai Savoir, trad. de P. Klossowski, Paris, Gallimard, ig67, \#354. 
Wittgenstein, répondrait sans doute que l'habileté de dire ce que nous sommes en train de faire ne révèle que la possibilité d'une rationalisation rétroactive de notre activité courante. Elle n'a pas à être fondée sur la recherche d'une cause mentale interne ${ }^{36}$.

Remarquez qu'en cherchant à expliquer la pensée de Heidegger, j'ai eu à parler d'activité plutôt que d'action, et cela du fait que Heidegger peut très bien soutenir que l'explication de l'action en termes de sujet et d'objet, évidente pour le sens commun, nécessite que les mouvements constituant l'action soient accomplis intentionnellement. Heidegger, par contre, n'essaie pas d'expliquer notre concept commun d'action, mais plutôt de faire place à une sorte de comportement, comme il le nomme, qui a été négligé tant par le sens commun que, a fortiori, par la tradition philosophique. La tradition s'est intéressée soit à rendre compte de l'action délibérée (Aristote), soit à déterminer la responsabilité morale (Kant). De tels projets se développent en se concentrant sur les croyances et les désirs conduisant à l'action (Davidson), ou à l'intention en action (Searle). Heidegger veut cependant développer une explication de notre préoccupation quotidienne courante et non délibérée. En laissant un tel comportement se montrer tel qu'il est en lui-même, Heidegger doit se libérer non seulement de la tradition, mais aussi de l'attention particulière que porte le sens commun à l'action délibérée. Comme le dit Heidegger :

Les prejjugés les plus dangereux et les plus tenaces, concernant la compréhension de lintentionnalité, ce ne sont pas les préjugés explicites sous formes de théories philosophiques, mais les préjugés implicites, ceux qui résultent de l'appréhension et de l'interprétation naturelle des choses par la compréhension quotidienne du Dasein. Or ce sont précisément eux qui sont les moins visibles et les plus difficiles à écarter ${ }^{37}$.

Heidegger soutient que les concepts du sens commun d'action et de conscience n'atteignent jamais la structure du mode le plus fondamental de notre comportement. À l'encontre de la tradition, il veut montrer que normalement, nous ne sommes pas thematiquement conscients de notre activité courante et quotidienne, et que lorsqu'apparaît une conscience thématique auto-

36. Pour une exposition plus détaillée de l'« en-vue-de » \« toward-wich »] dans l'activité chez Heidegger, voir mon live Being-in-the-world : A Commentary on Heidegger's Being and Time, Cambridge Mass., M.I.T. Press, Cambridge, 1991, chapitre 4.

37. Problemes fondamentaux., op.cit., p. 83 
référentielle, celle-ci présuppose un mode de conscience qui n'est ni thématique, ni auto-référentiel.

Dans la critique de l'explication kantienne de la perception qu'il développe dans les Problèmes fondamentaux de la phénoménologie, Heidegger suggère que la perception n'est pas non plus autoréférentielle, au sens de Grice, mais qu'elle est fondée sur une ouverture au monde qui n'est pas auto-référentielle. Tout comme l'action investie dans le monde, qui n'implique aucune expérience d'agir ni aucun état mental causant de façon auto-référentielle un mouvement corporel, la perception n'implique aucune expérience visuelle : je suis simplement fasciné et attiré par le spectacle du monde. Il remarque que « La perceptité l...l est, en un sens, objective, et, en un sens, subjective, et pourtant ni l'un ni l'autre $\gg^{38}$. C'est l'ouverture du Dasein au monde qui rend possible une expérience dérivée telle que regarder ou tenter de voir ; par exemple dans le cas extrême de l'examen de l'œil, cas qui pourrait probablement être décrit de façon à impliquer une expérience visuelle privée et causée par un objet ${ }^{39}$.

Être et Temps cherche à montrer que la majeure partie de l'activité quotidienne de l'homme, de son mode d'être propre, peut être décrite sans faire appel à la conscience auto-référentielle délibérée, et à établir comment une telle activité quotidienne peut ouvrir le monde et y découvrir des choses tout en ne comprenant

38. Ibid., p. 377.

39. On retrouve dans la Phénoménologie de la Perception de Maurice Merleau-Ponty, (Paris, Gallimard, 1976), une phénoménologie non mentaliste de la perception, parallèle à la phénoménologie heideggerienne de l'action de par sa recherche d'une rupture radicale avec la pensée sujet / objet. (Etant donné que Merleau-Ponty assista à Paris aux cours de Aron Gurwitsch sur l'explication par Heidegger du comportement dans les termes d'une « Gestaltstheorie » de la perception, il est possible de remarquer ici une influence directe de Gurwitsch sur Merleau-Ponty.) MerleauPonty développe l'idée d'une perception qui serait une pure ouverture au monde : "La perception d'autrui et le monde intersubjectif ne font problème que pour des adultes. L'enfant vit dans un monde quil croit d'emblée accessible à tous ceux qui l'entourent, il n'a aucune conscience de lui-même, ni d'ailleurs des autres, comme subjectivités privées, il ne soupçonne pas que nous soyons tous et qu'il soit lui-même limité à un certain point de vue sur le monde [J]. Il r'a pas la science des points de vue. Les hommes sont pour lui des têtes vides braquées sur un seul monde évident où tout se passe []. Piaget conduit l'enfant jusqu'á l'âge de raison comme si les pensées de l'adulte se suffisaient et levaient toutes les contradictions. Mais, en réalité, il faut bien que les enfants aient en quelque façon raison contre les adultes ou contre Piaget, et que les pensées barbares du premier âge demeurent comme un acquis indispensable sous celles de l'âge adulte, sil doit y avoir pour l'adulte un monde unique et intersubjectif $\gg$ (p. 407-408). 
aucune expérience, explicite ou implicite, de la séparation entre le mental et le monde des corps et des choses.

Mettre au jour la constitution existentiale du Dasein implique donc d'emblée une double tâche, qui en réalité n'en fait qu'une, celle d'interpréter de manière plus radicale les phénomènes de l'intentionnalité et de la transcendance. Avec cette tâche - prendre en vue, grâce à une conception plus originelle de l'intentionnalité et de la transcendance, une détermination fondamentale de l'existence du Dasein en général -, nous nous heurtons du même coup à un problème central, qui est resté jusqu'ici inconnu de toute la philosophie ${ }^{40}$.

Heidegger tentera de montrer que IIl l'intentionnalité sans contenu mental auto-référentiel caractérise le mode non contraint de l'activité quotidienne du Dasein, alors que l'intentionnalité des états mentaux est un mode dérivé, et [2] que ces deux modes de directionalité (transcendance ontique) présupposent une transcendance plus originaire : être-au-monde.

On verra que l'intentionnalité se fonde dans la transcendance du Dasein et qu'elle n'est possible que sur cette base ; tandis que la transcendance ne saurait en revanche s'expliquer à partir de l'intentionnalité ${ }^{4 \mathrm{I}}$.

Nous pouvons maintenant comprendre pourquoi, à la fin de la section 13, Heidegger prétend que « dans le connaître le Dasein acquiert un nouveau statut d'être à l'égard du monde qui est à chaque fois déjà dévoilé dans le Dasein ${ }^{42}$. Le mot «dans » ne doit pas, bien sûr, être ici compris au sens où le monde devrait se trouver, en tant que représentation interne, « à l'intérieur » du Dasein, mais plutôt au sens où le monde est découvert dans l'existence quotidienne du Dasein leveryday activity of Daseinl, dans l'être-là - au sein de cette activité qu'est l'existence, le mode d'être-à du Dasein.

\section{Université de Berkeley}

(Traduction francaise par J.-F. Campeau et P. Saudrais)

40. Problèmes fondamentaux, op. cit., p. 200.

4I. Ibid.

42. ÊET, op.cit., p. 97 ; SuZ, p. 62. 